\title{
High Incidence of Mitral and Tricupsid Regurgitation in Patients with Graves' Disease Detected by Two-Dimensional Color Doppler Echocardiography
}

\author{
Kumie Kage, Yuji Kira, Imao Sekine*, Fujiko Okabe, Takashi NaKaoka, Etsuo Hashimoto, \\ Masao Yamasaki**, Teruhiko OGITA and Etsuro Ogata*
}

\begin{abstract}
With two-dimensional (2D) color Doppler echocardiography, the cardiac and valvular function of 24 consecutive patients with a history of Graves' disease (17 were hyperthyroid and 7 were euthyroid at the time of the examination) were evaluated. The incidences of mitral regurgitation (MR), tricuspid regurgitation (TR) and MR plus TR were significantly higher in the patients with Graves' disease than in the age-matched control group of patients without this disease. In the patients who had signs of congestive heart failure (CHF) while they were hyperthyroid, a significantly higher incidence of severe TR was observed. This is the first report of a $2 \mathrm{D}$ color Doppler echocardiography study on the incidences of TR and/or MR in patients with Graves' disease. Our data indicate that in Graves' disease valvular dysfunction can be caused by systemic disorders and that severe TR is a possible risk factor for CHF.
\end{abstract}

(Internal Medicine 32: 374-376, 1993)

Key words: hyperthyroidism, valvular heart disease, risk factor for $\mathrm{CHF}$

\section{Introduction}

Graves' disease complicated with CHF has therapeutic disadvantages since the use of $B$-adrenergic blockers can aggravate CHF. A high cardiac output state and an increased incidence of supraventricular tachycardia, especially atrial fibrillation (Af) (1), are well-known cardiovascular complications of Graves' disease, whereas the valvular complications are less well recognized. There are a number of studies on mitral valve prolapse (MVP) in hyperthyroidism (2), but only a few case reports $(3,4)$ discuss the presence of valvular regurgitation in hyperthyroid patients. The significance of valvular regurgitation remains unclear because there have been no studies examining the incidence of valvular regurgitation as a complication of Graves' disease.

Recent advances in cardiac imaging by 2D color Doppler echocardiography have made it possible to detect and accurately evaluate valvular disorders. By utilizing this apparatus, we studied the prevalence of MR and/or TR in patients with documented Graves' disease and assessed the significance of valvular regurgitation in the clinical manifestation of this disease.

\section{Methods}

Between 1989 and 1991,24 consecutive patients with Graves' disease (aged 21 to 77 years, mean \pm SD: $49.0 \pm 7.1$ years) were studied by 2D color Doppler echocardiography to evaluate their cardiac and valvular function. While 17 patients were studied before or during the early phase of their treatment for hyperthyroidism, 5 suffered from chronic heart failure. [CHF was defined as the presence of an increased cardiothoracic ratio on the chest radiogram, pretibial pitting edema and dyspnea.] Seven patients had been treated with propylthiouracil or methimazole and were already euthyroid for $14.2 \pm 13.2$ months (range: 1 to 36 months) when the study was undertaken. Though three of these seven patients had been in CHF prior to the treatment, they improved at the time of the study. None of the patients had a history of cardiovascular or pulmonary disease before the diagnosis of Graves' disease was made. Hyperthyroidism due to Graves' disease was diagnosed by the presence of the typical symptoms, physical findings and serum concentrations of TSH measured using an immunoradiometric assay kit (DAINABOT, Japan) and free serum T4 concentrations (Amersham, Japan). The criteria for thyrotoxicosis were

\footnotetext{
From the Cardiovascular Laboratory, the Fourth Department of Internal Medicine, University of Tokyo School of Medicine, *Cancer Institute Hospital, Tokyo and **Mitsui Memorial Hospital, Tokyo

Received for publication June 24, 1992; Accepted for publication April 19, 1993

Reprint requests should be addressed to Dr. Kumie Kage, the Fourth Department of Internal Medicine, University of Tokyo, 3-28-6 Mejirodai, Bunkyo-ku, Tokyo 112, Japan
} 


\section{High Incidence of MR \& TR in Graves’ Dis.}

as follows: a serum TSH suppressed below $0.5 \mu \mathrm{U} / \mathrm{ml}$ and a free serum T4 higher than $2.0 \mathrm{ng} / \mathrm{dl}$.

The control group consisted of 76 consecutive individuals without known cardiovascular or pulmonary diseases by history (aged 20 to 82 years, mean \pm SD: $49.2 \pm 15.3$ years), who were evaluated by echocardiography during the course of a routine check-up. M-mode and 2D echocardiography for the evaluation of chamber size and ventricular function, and a color Doppler study for the detection of valvular regurgitation were performed as described (5) with a commercially available system (SSA 160A Toshiba, Japan). Only valvular retrograde flow throughout the systolic period was scored as positive regurgitation, and the severity of the valvular regurgitation was assessed visually and semiquantified into three grades from mild to severe. In order to estimate the grade of regurgitation on the basis of distribution of the regurgitant jet detected within the atrium, the distance from the valvular orifice to the bottom of the atrium was divided into three equal length. Thus, mild regurgitation was detected if the jet reached the first portion; moderate regurgitation when the jet reached the second portion; severe regurgitation when the jet occupied all over the atrium. The results were assessed by more than three independent observers and all of them agreed on the assessments. For statistical analysis, two sample $t$-test and $\chi$-square test were utilized.

\section{Results}

There were no significant differences in left ventricular (LV) size and LV function between the group of patients with Graves' disease (the GD group) and the control group. Af was recognized in seven patients in the GD group but in none in the control group (Table 1). The incidences of MR, TR, and MR plus TR were significantly higher in the GD group than in the control group. The prevalence of MVP was higher in the GD group than in the control group, but it was not statistically significant. MVP was present in $25 \%$ of the GD patients who had MR (Table 2). Overt tricuspid valve prolapse (TVP) was observed in only three GD patients. There were no significant differences in the LV dimension and ejection fraction between patients with MR and those without MR in the GD group (data not shown).

Table 1. Patient Characteristics

\begin{tabular}{cccc}
\hline & Graves' disease & Control & P value \\
\hline No. of patients & 24 & 76 & \\
Sex M:F (\% of F) & $2: 22(92 \%)$ & $39: 37(49 \%)$ & \\
Age (y.o.) & $49.0 \pm 16.2$ & $49.2 \pm 15.3$ & NS \\
Af & $7(29 \%)$ & 0 & $<0.01$ \\
& & & \\
LVDd (mm) & $49.2 \pm 7.1$ & $48.4 \pm 6.2$ & NS \\
LVDs (mm) & $31.5 \pm 6.0$ & $32.6 \pm 7.2$ & NS \\
EF (\%) & $65.4 \pm 6.9$ & $63.9 \pm 7.0$ & NS \\
\hline
\end{tabular}

LVD: left ventricular dimension, d: dialtolic, s: systolic, EF: ejection fraction.
Eight of 24 patients appeared to have histories of CHF while they were hyperthyroid (Table 3 ). Severe TR was observed only in the GD patients with signs of CHF. Three of these $8 \mathrm{CHF}$ patients had both Af and severe TR. Severe MR was observed in 2 patients with and 2 without signs of CHF. There were no significant differences in the ages between the GD patients with and without CHF. Similarly high incidences of MR, TR, and MR plus TR were observed in the GD group even when the patients had become euthyroid after treatment (Table 4).

Table 2. The Prevalence of Valvular Disorders

\begin{tabular}{|c|c|c|c|}
\hline & Graves' disease & Control & \\
\hline No. of patients & 24 & 76 & \\
\hline MR & $17(71 \%)$ & $2(3 \%)$ & $<0.01$ \\
\hline TR & $15(63 \%)$ & $12(16 \%)$ & $<0.01$ \\
\hline Mild & 8 & 10 & NS \\
\hline Moderate & 3 & 2 & NS \\
\hline Severe & 4 & 0 & $<0.01$ \\
\hline $\mathrm{MR}+\mathrm{TR}$ & $14(58 \%)$ & 0 & $<0.01$ \\
\hline MVP & $6(25 \%)$ & $11(14 \%)$ & NS \\
\hline MR+MVP & $6(35 \%$ of $M R)$ & 0 & NS \\
\hline
\end{tabular}

MR: mitral regurgitation, TR: tricuspid regurgitation, MVP: mitral valve prolapse.

Table 3. Incidence of CHF in Patients with Graves' Disease with and without Severe TR and Af

\begin{tabular}{ccc}
\hline & CHF (+) & CHF (-) \\
\hline No. of patients & 8 & 16 \\
Severe TR & 4 & 0 \\
Af & 4 & 3 \\
Severe TR+Af & 3 & 0 \\
Neither severe TR nor Af & 4 & 12 \\
Severe MR & 2 & 1 \\
Severe MR+Af & 2 & 1 \\
\hline
\end{tabular}

CHF: congestive heart failure, MR: mitral regurgitation, TR: tricuspid regurgitation, Af: atrial fibrillation.

Table 4. Incidence of Valvular Regurgitation in Patient with Graves' Disease as a Function of Thyroid State

Thyroid function at the time of evaluation

\begin{tabular}{cccc}
\hline & Hyperthyroid & Euthyroid & P value \\
No. of patients & 17 & 7 & \\
Total of MR* & 12 & 5 & NS \\
Only MR & $2(12 \%)$ & $1(14 \%)$ & NS \\
Only TR* & $1(6 \%)$ & 0 & NS \\
MR+TR & $10(59 \%)$ & $4(57 \%)$ & NS \\
MR+MVP* & $3(18 \%)$ & $3(43 \%)$ & NS \\
\hline
\end{tabular}

*Abbreviations are the same as in Table 2. 


\section{Discussion}

This is the first report on the use of 2D color Doppler in which the incidence of MR and/or TR in patients with Graves' disease was studied. A significantly higher incidence of valvular regurgitation was observed in the patients with Graves' disease than in the control patients. Although the incidences of MR and TR in our control group were lower than those in a previous report (6), the prevalences of MR and TR were still significantly higher in the GD group than in that previously reported control group. We found that $71 \%$ of our GD patients had MR. A previous study reported that the incidence of MR in hyperthyroid patients was only $6.2 \%$ (7). However, the authors used phonocardiography to detect MR, which is considerably less sensitive than 2D echocardiography as used in this study. The discrepancy of the incidences in both reports may thus be attributed to the differences in the method for detecting MR.

MR in hyperthyroidism has been inferred to be a consequence of mitral annular dilatation due to left ventricular dilatation or that of MVP. However, the present data suggest that another causative mechanism is involved, since we found no correlation between the incidence of MR and LV dimension and less than half of the GD patients with MR possessed concomitant MVP .

The incidence of TR was also higher in the GD group than in the control group. In particular, severe TR was observed only in the GD group, whereas mild to moderate TR, which is hemodynamically insignificant, was observed in both groups. We cannot attribute the mechanism of TR to TVP in the GD group, because only $20 \%$ of patients with TR had TVP. Moreover, signs of CHF were observed more frequently in the GD patients with severe TR than in those without it. Although there were 2 patients with severe MR, who had signs of CHF, they also had severe TR. Half of the patients with signs of CHF exhibited Af. These findings suggest that severe TR, and possibly MR, could have a deleterious effect in addition to Af on the cardiovascular system in GD patients. It should be noted that these valvular abnormalities persist for a couple of years after the state of thyrotoxicosis is controlled. Thus, the existence of a direct and permanent effect of the thyroid hormone on the mitral and tricuspid valves cannot be denied.

In conclusion, we suggest that it is important to examine for TR and MR in patients with Graves' disease not only because of its high prevalence but also because of its clinical significance as a risk factor for CHF.

\section{References}

1) Hurst JW, Schlant RC, Rackley CE, et al. The Heart (7th ed. Hurst JW) 1990, p.463, p.1498. McGraw-Hill, New York.

2) Channick BJ. Hyperthyroidism and mitral valve prolapse. N Engl J Med 305: 497, 1981.

3) Dougherty MJ, Craige E. Apathetic hyperthyroidism presenting as tricuspid regurgitation. Chest 63: 767, 1973.

4) Reynolds JL, Woody HB. Thyrotoxic mitral regurgitation. Am J Dis Child 122: 544, 1971.

5) Henry WL, DeMaria A, Weyman AE, et al. Report of the American Society of Echocardiography Committee on Nomenclature and Standards in Two-dimensional Echocardiography. Circulation 62: 212, 1980.

6) Yoshida K, Yoshikawa J, Fukuya T, et al. Color Doppler evaluation of valvular regurgitation in normal subjects. Circulation 78: 840, 1988.

7) Yamaguchi T. A study of mitral valve dysfunction in hyperthyroidism using phonocardiography. The Tokyo Journal of Medical Sciences 93: $179,1986$. 